\title{
Quantitation of Thin-Layer Chromatograms with an Apple II Computer-Based Videodensitometer
}

\author{
THOMAS S. FORD-HOLEVINSKI AND NORMAN S. RADIN \\ Mental Health Research Institute, University of Michigan, Ann Arbor, Michigan 48109
}

Received July 1, 1985

\begin{abstract}
An inexpensive, high-speed densitometer made from an Apple II computer, a black-and-white video camera, and an image digitizing board is described. By supplementing the computer with a very fast coprocessor, one can obtain a measurement of a typical thin-layer spot in 30-40 s. Only minimal electronic expertise is required for assembling the system from the commercially available components. Coefficients of variation for multiple readings of a single 500-ng spot of charred lipid were about $0.5 \%$. For lipid spots weighing 250 to $2000 \mathrm{ng}$, a second-order relationship between weight and density reading was obtained with a correlation coefficient of 0.998 . (c) 1985 Academic Press, Inc.

KEY WORDS: thin-layer chromatography; densitometry; lipid determination; computerized image scanning.
\end{abstract}

Spots and bands of light-absorbing, fluorescent, or chemiluminescent materials on transparent or translucent supports (thin-layer plates, radioautograms, paper chromatograms, electrophoresis gels, etc.) are ordinarily quantitated with a scanner. This is typically a mechanical device which moves the support past a light sensor, such as a photomultiplier or photocell. For light-absorbing spots, a light source is placed under or above the spot (for absorbance or reflectance measurements) and a mechanical slit is used to define the beam.

About 10 years ago, a research group in Hungary undertook a new approach to the problem by using a video camera and a large computer to measure the darkness of a spot $(1,2)$. Advantages of a videodensitometer are that no slit is necessary, an entire field of spots can bc measured with a single scan, a mechanical support mover is unnecessary, and resolution up to any degree (within the limits of light scattering) can be attained by choosing an appropriate lens. Several researchers have adapted this approach and its popularity has been increasing as small computers have increased in power (3-6). More precise and reliable, but more expensive, charge-coupled devices are beginning to replace the common Vidicon-type camera.

We recently reported the construction of an inexpensive videodensitometer based on a black-and-white video camera, an Apple II computer, and other commercially available components (7). We used a fluorescent lamp under the TLC plate as light source, with the camera mounted overhead. This system was used to analyze charred lipid spots on TLC and HPTLC ${ }^{1}$ plates. We have modified the device and significantly improved both its precision and the speed at which it operates. This was done by adding a high-speed coprocessor board to work in conjunction with the computer. The decreased time required for a complete measurement, now $30-40 \mathrm{~s}$, minimizes the problem of drift in the camera and light source. The computer program that operates the device has been elaborated to display additional visual information and to print out a permanent record of the reading, as well as textual identifying information. The resultant system produces a relatively high degree of

\footnotetext{
'Abbreviation used: HPTLC, high-performance thinlayer chromatography.
} 
precision at low cost with samples weighing 250-2000 ng.

\section{EXPERIMENTAL SECTION}

Computer components. In both the original and the improved systems a Dithertizer II plug-in card (Computer Stations, Inc., St. Louis, Mo.) is used in the computer to convert an analog video signal into a binary image on the Apple II's high-resolution screen. The previously reported device (7) utilized only the Apple II's onboard 6502 microprocessor to determine which picture elements ("pixels") were uniquely lit at each of the 256 possible threshold levels. This was done by subtracting the image obtained at one threshold from the image generated at the previous, higher level. The new threshold value was subsequently stored in a memory expansion card in 1-byte locations which corresponded to the location of those unique bits on the high-resolution screen. This generated an 8-bit representation of the plate scanned and also its density information.

We have now replaced the memory expansion card with a Dtack Grande board and Stuffer interface (Digital Acoustics, Santa Ana, Calif.). This board contains a Motorola 68000 microprocessor, running at an effective rate of $10 \mathrm{MHz}$, and 128 kbytes of memory which are not directly accessible to the Apple's microprocessor. In the new system each of the images generated by the Dithertizer II is transferred to the 68000 board by direct memory access where it is manipulated as before to generate the final 8-bit data representation.

A 500-W voltage regulator and filter (Sola Electric Mini/Micro Computer Regulator) is used to power the entire system. The system is turned on at least $2-3 \mathrm{~h}$ before use to stabilize all components.

A second monitor is used to exhibit the normal video picture, enabling the user to move the TLC plate to center the next spot under an $\mathrm{X}$ marked on the face of the monitor. The movement is done while the computer performs calculations on the previously cap- tured image. The camera and fluorescent light box are stationary, minimizing variations in the uniformity of the light source. A weakness in videodensitometers arises from nonuniformity of the light-sensitive elements in the detector, so that the observed darkness of any spot depends on its location in the image field. It is possible to store corrections for this nonuniformity in the computer memory, but more memory and a much more expensive camera are needed for this approach. We have eliminated this problem by examining only a single TLC spot at a time, in the same detection field.

The total cost of the densitometer is now about $\$ 2760$, excluding the cost of the Apple computer (IIe or II + ) and printer. The computer is, of course, available for other applications and the Dtack board, for which various languages are available, can be used to greatly increase the speed of some other programs. The memory in the board can be expanded to 1 Mbytc.

Software. The software required to run this system has been altered to provide more visual information to the user. The program displays (i) an outline of the spot that was scanned, (ii) a rectangle surrounding the spot to be analyzed, (iii) the curves depicting the digital values of each video scanline within the rectangle, (iv) the baseline drawn for each curve in the scanline, (v) the edges of the spot scanned, as calculated by a slope-sensing algorithm, and (vi) a curve showing the maximal value of each scanline in the rectangle (Fig. 1). The rectangle dimensions are set by the user with a joystick after a scan of the largest spot. Once set, subsequent spot measurements utilize the rectangle as a default frame.

The initial digital display of the spot, used to guide the operator in designating the rectangle to be scanned, is derived by turning on all the pixels with intensity values between two of the lower thresholds. This produces an outline image of the spot which is slightly smaller than the computer-calculated edges of the spot.

At the left side of the screen is displayed the curve of pixel density values as each line in 


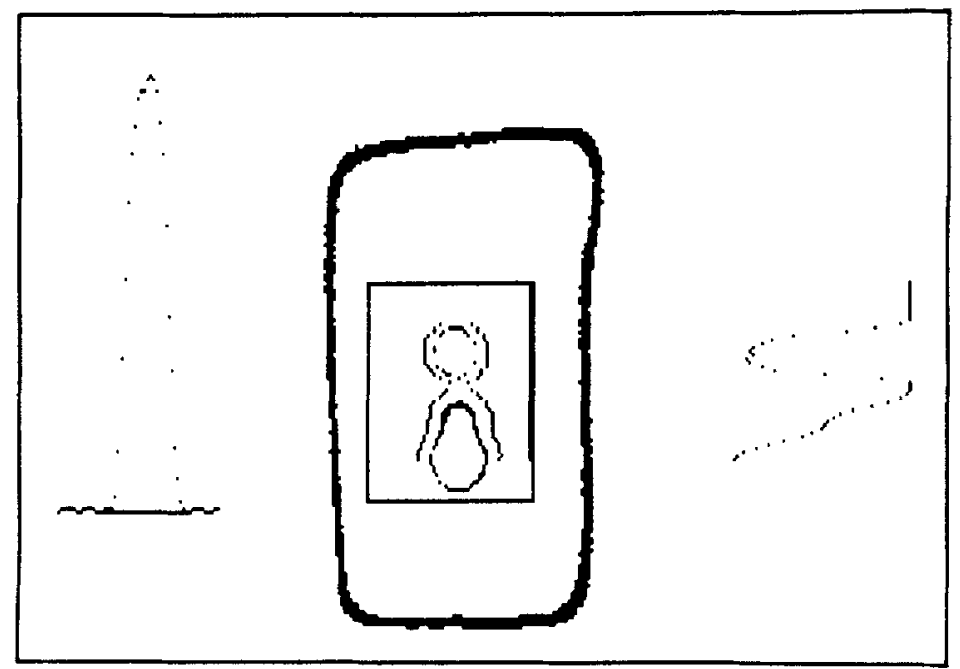

FIG. 1. Computer monitor display during the calculation stage of the densitometer program. In the center of the display is the rectangle which defines the region to be quantified, as well as the outlines of two spots whose image had been captured a few seconds ago. (Normally one sets the rectangle to enclose just one spot.) The dots surrounding the spot, inside the rectangle, are the true edges of the spot, located by the slopesensing algorithm. Surrounding the rectangle is the outline of the viewing window made by cutting a rectangular hole in a sheet of black paper, which sits on the light box. The left side of the screen shows the pixel intensity values of the scanline just examined as well as its calculated baseline. The right side of the screen shows a plot of the maximal pixel darkness values of each scanline thus far measured.

the rectangle is examined. The examination for the left edge of the spot consists of comparing the density values of two pixels spaced a predefined distance apart (pixels 4 and 8 in Fig. 2). If the comparison shows that the right pixel is as dark as or paler than the left one, a new pair of pixels is examined. If this pair bears the same relationship, additional pairs are compared. If the new right pixel is darker by a preset value than the new left pixel, the center pixel between them is declared to be the left edge of the spot. A similar calculation is carried out on the right side of the scanline to locate the right edge of the spot.

A baseline is then drawn on the displayed curve between the two edges. The pixel intensities between them, after subtracting the baseline values, are summed to give the total intensity for that scanline. This value is added to the cumulative total for all thc scanlines in the rectangle. When all the scanlines have been analyzed, the total pixel values are printed out by the computer. Like the "counts" of electronic integrators, these are arbitrary units which must be compared on a standard curve against standard spots.

The maximal value for the peak in each scanline is displayed as a curve on the right side of the screen (Fig. 1). This is helpful in determining whether the bottom and top lines in the scanned rectangle were chosen appropriately. Ideally, assuming that nearby TLC spots are adequately separated from the scanned spot, a flat baseline should be seen at the top and bottom of this curve. The same is true of the successive scans shown at the left side of the screen. In Fig. 1, a second spot has deliberately been enclosed within the rectangle to illustrate how this display could be used to delineate one desired spot.

The software was written in 6502 and 68000 assembly language, as well as Applesoft BASIC, which was compiled for higher speed with the TASC Compiler (Microsoft Corp., Bellevue, Wash.).

Chromatographic materials. TLC plates, commercially coated with silica gel 60 (EM Science, No. 5763), were cut to $10 \times 10 \mathrm{~cm}$ 


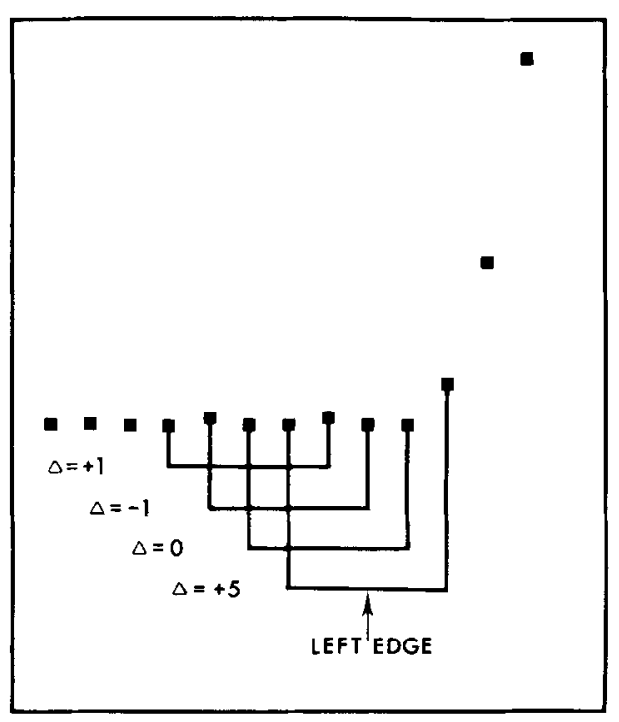

FIG. 2. An enlargement of the left side of the scanline shown in Fig. 1 showing the slope-sensing calculation. Each square represents a pixel and the height of the pixel corresponds to its darkness value. The lower lines show four successive pixel comparisons and the final decision (marked by an arrow) locating the left edge of the spot on that scanline.

and samples were applied with the Transpot HPTLC Contact Sample Applicator (details to be published). The plates were developed first with chloroform, and then with chloroform-methanol-water containing ammonium hydroxide and $\mathrm{KCl}$. A glycolipid, glucosyl ceramide, and a phospholipid, phosphatidyl ethanolamine, were used as the test samples. A copper sulfate-phosphoric acid charring reagent (8) was used to make the spots visible. The plate was covered with a clear glass plate and stored in a desiccator to stabilize the brownish-gray spots.

\section{RESULTS AND DISCUSSION}

Speed. The use of the 68000 board decreased the time required to generate the 8bit image from over $2 \mathrm{~min}$ to approximately $12 \mathrm{~s}$. This is due to the speed of the Motorola 68000 microprocessor, its ability to access directly each byte in the 8-bit data representation (the 6502 had to use bank selection in the expanded memory card), and the fact that at many times both processors are working simultaneously. The time required to calculate and display the scanning curves and final "counts" is 18-28 s, depending on the size of the spot and surrounding rectangle. During the calculation period one can move the TLC plate to bring the next spot into position for the next analysis. The total measurement time of 30-40 s compares well with other available scanners, some of which require multiple scans to evaluate the entire spot, or which require manual examination of the integrator's pen recordings.

Reproducibility. It was found that significant drift occurred during the first $2-3 \mathrm{~h}$ after the equipment was turned on, due in part to downward voltage drift in the power regulator's output (measured with a power-line voltmeter). After stabilization, the regulator fluctuated within a range of about $0.1 \mathrm{~V}$. While this represented a distinct improvement over the previous use of unstabilized power (7), repeated measurements of a single spot showed that there were still occasional drifts in the readings. These may originate in shifts in the output of the fluorescent lamp and one could reduce this source of drift by substituting a power-controlled tungsten lamp.

When a $0.5-\mu \mathrm{g}$ glucosyl ceramide spot was charred and read 42 times in succession over a period of $40 \mathrm{~min}$, the means calculated for successive groups of seven readings were 38241, 38007, 37969, 37853, 37838, and 37595. This particular run showed a downward drift of 646 density units $(1.7 \%)$. The CV's were $0.51,0.45,0.73,0.65,0.54$, and $0.76 \%$, respectively. These values compare quite favorably with other densitometric methods.

Smaller spots showed greater variability. When a $0.25-\mu$ g glucosyl ceramide spot was read 24 times in succession, the observed mean was 20035 and the $\mathrm{CV}$ was $0.95 \%$ (equivalent to $2.5 \mathrm{ng}$ ). In this run there was no noticeable drift.

These data show that the videodensitometer produces good precision and that the effect of drift can be reduced considerably by rapidly reading all the spots of a single compound on one plate (standards and unknowns).

Spot-to-spot resolution. This is affected by 
plate heterogeneity, image size on the screen, $f$-stop value on the camera lens, and the number of thresholds that the image digitizer can produce. Using a 15 -W fluorescent bulb in the light box and a lens aperture of 5.6, we obtain about 155 levels of gray for charred spots on standard $0.25-\mathrm{mm}$-thick silica gel plates. This range of gray values is somewhat superior to the 64 levels offered by other presently available commercial image digitizers usable in the Apple II computer.

Using a closeup lens on our camera, we obtain a spot size on the screen corresponding to $30-35$ scanlines for a $1-\mu \mathrm{g}$ spot of $3 \mathrm{~mm}$ in the middle of a $10-\mathrm{cm}$ plate. Thus the limit of separation between two adjacent spots is about $0.1 \mathrm{~mm}$. This degree of spatial resolution is superior to some of the mechanooptical slittype TLC scanners available. The recently offered laser scanners produce high resolution but require multiple adjacent scans or very precise band shapes because the beam is not wide enough to visualize an entire spot (9).

Standard curve. For a typical plate containing spots weighing 250 to $2000 \mathrm{ng}$, the range of readings (in absolute units) was about 25,064 to 112,180 for glucosyl ceramide and 10,000 to 57,440 for phosphatidyl ethanolamine. Good linearity was observed in the more restricted range of 400 to $1000 \mathrm{ng}$, with a slope of 0.017 for the glycolipid and 0.036 for the phospholipid. The correlation coefficients for the two (derived from 10 of the 14 spots) were 0.9979 and 0.9975 . This range of linearity could be expanded to 250 to $2000 \mathrm{ng}$ by using a plot that is second order with respect to readings, which gave slightly higher correlation coefficients for the two lipids. The correlation coefficients cited here include the experimental variations in spot application, solvent development, charring, and densitometry. Papers on TLC quantitation systems have reported a variety of relationships between readings and weights; most seem to find a nonlinear relationship and greater variability.

While the dynamic range of the TLC system used in this test is not as great as one would like, it is nevertheless useful. It may be that other samples and spot detection reagents would yield a wider range. It should be noted that our use of round spots, made with the Transpot applicator, allows the application of 13 to 14 spots on a $10 \times 10-\mathrm{cm}$ plate; this is more efficient than preadsorbent-coated plates, which accomodate fewer spots.

One can anticipate application of the videodensitometer to other transparent or semitransparent samples, such as radioautograms and immunoassay colored spots, and to fluorescent bands in gels. Light-emitting spots ought to show a wider dynamic range than light-absorbing spots.

The system described in this paper can be assembled with minimal electronic or mechanical expertise. The software and details of construction are available by application to the authors.

\section{ACKNOWLEDGMENTS}

We are indebted to Inez Mason for her careful TLC operations, measurements, and calculations. This research was supported in part by National Institutes of Health Grants HD-018645, EY-05947, and training grant MH15794.

\section{REFERENCES}

1. Devenyi, T. (1976) Acta Biochim. Biophys. Acad. Sci. Hung. 11, 1-10; Chem. Abstr. (1977) 86, 1901.

2. Devenyi, T., and Pogor, S. (1980) Hung. Sci. Instrum. 50, 3-8; Chem. Abstr. (1981) 95, 111172.

3. Gianelli, M. L., Burns, D. H., Callis, J. B., Christian, G. D., and Andersen, N. H. (1983) Anal. Chem. 55, $1858-1862$.

4. Rees, D. D., Fogarty, K. E., Levy, L. K., and Fay, F. S. (1985) Anal. Biochem. 144, 461-468.

5. Aycock, B. F., Weil, D. E., Sinicropi, D. V., and Mcllwain, D. L. (1981) Comput. Biomed. Res. 14, 314-326.

6. Gianelli, M. L., Callis, J. B., Andersen, N. H., and Christian, G. D. (1981) Anal. Chem. 53, 1357 1361.

7. Ford-Holevinski, T. S., Agranoff, B. W., and Radin, N. S. (1983) Anal. Biochem. 132, 132-136.

8. Touchstone, J. C., Levin, S. S., Dobbins, M. F., and Carter, P. J. J. (1981) High Resolut. Chromatogr. Commun. 4, 423-424.

9. Goppelt, M., and Resch, K. (1984) Anal Biochem. 140, $152-156$. 